\title{
Strates
}

STRATES Matériaux pour la recherche en sciences sociales

6 | 1992

La question de l'environnement : naissance d'un débat en Pologne

\section{Catastrophe écologique, fragilité du mouvement vert : la voïvodie de Katowice}

Marek S. Szczepanski

\section{(2) OpenEdition \\ Journals}

Édition électronique

URL : http://journals.openedition.org/strates/2803

DOI : $10.4000 /$ strates.2803

ISSN : $1777-5442$

Éditeur

Laboratoire Ladyss

Édition imprimée

Date de publication : 31 mars 1992

ISSN : 0768-8067

Référence électronique

Marek S. Szczepanski, « Catastrophe écologique, fragilité du mouvement vert : la voïvodie de

Katowice », Strates [En ligne], 6 | 1992, mis en ligne le 22 novembre 2007, consulté le 08 septembre

2020. URL : http://journals.openedition.org/strates/2803; DOI : https://doi.org/10.4000/strates.2803

Ce document a été généré automatiquement le 8 septembre 2020.

Tous droits réservés 


\title{
Catastrophe écologique, fragilité du mouvement vert : la voïvodie de Katowice $^{1}$
}

\author{
Marek S. Szczepanski
}

1 En Pologne, les mouvements sociaux revendicatifs ayant pour but est de limiter les processus de dégradation de l'environnement naturel et de rétablir un équilibre relatif dans les régions touchées par une catastrophe écologique se trouvent encore in statu nascendi. Actifs dans les vingt- sept régions du pays considérées comme écologiquement menacées (qui couvrent $55200 \mathrm{~km}^{2}$ et comptent 12300000 habitants) et dans les régions écologiquement sinistrées, ils présentent un faible degré d'organisation dont les causes sont multiples, la plus importante étant liée au caractère coercitif de l'État du socialisme réel.

2 Cet État communiste - et les fonctionnaires qui le desservaient - était une institution totalitaire, qui avait l'ambition de contrôler et de réglementer les comportements des individus et de surveiller tous les mouvements sociaux, y compris les mouvements écologiques. On considérait que concéder à des mouvements plus ou moins indépendants un droit de regard sur la politique d'environnement aurait affaibli le pouvoir central.

3 L'inconsistance des mouvements écologistes est aussi liée directement au manque de conscience écologique de la population, car beaucoup d'informations sur l'état de l'environnement comportaient une clause "secret» et leur accès était interdit au public. Cette difficile situation des mouvements écologistes est encore aggravée par des conflits opposant leurs leaders, au niveau régional ou local: le parti polonais des «Verts » est ainsi divisé par les ambitions des leaders de ses nombreuses fractions.

La voïvodie de Katowice, région écologiquement sinistrée

4 La voïvodie de Katowice est la région la plus industrialisée de Pologne. Le déséquilibre de l'environnement naturel y est tel que les députés du conseil de voïvodie ont considéré en 1988 cette région comme zone écologiquement sinistrée. Dans de nombreux travaux réalisés par des organisations internationales, la voïvodie est mentionnée 
parmi les cinq régions du monde dont l'environnement naturel est le plus dégradé. Les villes de la région silésienne figurent parmi les plus gravement atteintes et l'une de ces villes - Zabrze - est généralement considérée comme l'agglomération écologiquement la plus délabrée du monde.

5 La voïvodie couvre $6650 \mathrm{~km}^{2}$, ce qui ne représente que $2,1 \%$ de la superficie de la Pologne. Elle est pourtant habitée par près de quatre millions d'habitants, soit $10,5 \%$ de la population polonaise. Dans cette région se trouvent $15 \%$ de l'industrie nationale et 238 usines qui sont particulièrement nuisibles à l'environnement naturel. On extrait ici $98 \%$ du charbon du pays, on produit $50 \%$ de l'acier, $47 \%$ des produits laminés et la totalité du zinc et du plomb. En 1990, cette voïvodie assure ainsi $25 \%$ du revenu national et $20 \%$ des produits d'exportation. Cette hyperconcentration de l'industrie a eu des conséquences écologiques dramatiques : sont émis dans cette région $28 \%$ des gaz et $22 \%$ des poussières industrielles, et sont rejetés dans les eaux de surface $22 \%$ des effluents d'égouts non purifiés. On y produit $52 \%$ de tous les déchets industriels du pays, soit plus d'1,5 milliard de tonnes. Le gigantesque dépôt d'ordures s'agrandit chaque année de 100 millions de tonnes et dévore 350 hectares de sol utilisable. Si l'on répandait tous ces déchets sur la surface de la voïvodie en une couche uniforme, sa hauteur atteindrait plus de 1,70 mètres.

6 Les normes nationales de condensation des substances toxiques dans l'air, dans l'eau et dans le sol y sont partout largement dépassées. En plusieurs lieux, on a enregistré une concentration équivalant à mille fois la norme pour le plomb, et à plusieurs centaines de fois la norme pour le bismuth, le zinc, le cadmium, le cuivre, le fer, le calcium, le chlore, l'aluminium et le mercure. On peut y extraire jusqu'à cinq grammes de plomb dans un kilogramme de terre.

7 Dans cet environnement dégradé vit et travaille une population qui subit les conséquences désastreuses de cette situation dramatique. Un million de personnes, chaque jour, demeurent dans des lieux où les normes écologiques les plus " tolérantes " sont ainsi dépassées. On ne s'étonne donc pas que leur état de santé soit très mauvais. Ici, on meurt trois fois plus souvent à cause de la tuberculose que dans le reste du pays, on est plus souvent atteint par le cancer $(+10 \%)$, le diabète $(+50 \%)$ et les maladies cardiaques $(+20 \%)$. Près de $45 \%$ des femmes enceintes subissent des pathologies de la grossesse et $10 \%$ des nouveaux-nés naissent avant terme. $15 \%$ des jeunes enfants ont des troubles de la croissance, et ils sont sujets à des maladies des voies respiratoires quatre fois plus souvent que les enfants des autres régions. La mortalité des nouveauxnés, dans cette voïvodie, est accablante. En Pologne, en 1990, on enregistre une mortalité de 17 décès pour 1000 naissances, tandis qu'ici on en dénombre 20. Dans sa partie la plus dégradée, on compte même 30 décès. Il $\mathrm{y}$ a des villes et des quartiers où ces taux sont encore plus effroyables, par exemple dans le quartier sidérurgique et minier Rozbark de la ville de Bytom, où on compte 52 décès pour 1000 naissances, ou dans des villes comme Zabrze, Ruda Slaska, Chorzow, où ce taux est à peine moins élevé.

8 En 1989, les scientifiques de l'Académie de Médecine de Silésie ont mené des recherches sur l'impact de la dégradation de l'environnement sur la santé de la population. Une classification a ainsi été établie, en attribuant à chaque voïvodie un point pour chaque dépassement de la norme écologique nationale. Pour la voïvodie de Katowice, cet indicateur atteint 3000 points (pour Zabrze, 5600 points), alors qu'il n'est que de 950 pour la voïvodie de Legnica, la deuxième sur la liste, 790 pour la voïvodie de Cracovie, 
530 pour celle de Tarnobrzeg, 430 pour Opole, 350 pour Bydgoszcz, 330 pour Szczecin, 250 pour Gdansk, 240 pour Lodz. Cette classification montre à quel point la santé de la population est menacée dans la voïvodie de Katowice, l'ampleur de cette menace n'étant pas comparable à celle des autres régions.

9 La situation écologique catastrophique et l'état de santé déplorable de la population silésienne inclinent à se demander si des actions visant à assurer la protection de l'environnement ont été entreprises par les autorités locales et régionales, les habitants eux-mêmes, ou les organisations et mouvements politiques. Le pouvoir communiste de la voïvodie a investi avec détermination dans le développement de l'industrie lourde. Les actions qui ont abouti à une hyperconcentration des technologies polluantes avaient leurs raisons d'être: les élites locales du parti et de l'administration renforçaient ainsi leur position politique et économique dans le pays. À cette époque, détenir des industries dans la région que l'on administrait constituait non seulement un acte de fétichisme envers les " géants » industriels, mais avait aussi une signification symbolique, et déterminait effectivement le prestige et la position de l'élite régionale dans la configuration du pouvoir central. Il n'est donc pas étonnant qu'on ait construit dans cette voïvodie la plus grande forge, la plus grande laiterie, et les deux plus grandes mines d'Europe. Il y a peu de temps, on envisageait encore d'y construire une importante raffinerie de pétrole et d'y creuser une autre grande mine.

10 Cette politique d'investissements effrénés a eu de graves conséquences. Les autorités régionales ont aisément toléré les dépassements des normes écologiques des usines "prioritaires» (tel était leur nom). Avec le consentement implicite ou explicite des autorités, l'industrie détruisait régulièrement la forêt, où de nouvelles implantations étaient effectuées. Elle dévorait les terrains agricoles où l'on construisait des chantiers. Et on bâtissait des quartiers d'habitation dans les zones abritant de grands complexes industriels et des cokeries, en assurant qu'elles «amélioraient l'état de l'environnement». Ces décisions absurdes ont provoqué une aggravation importante de l'état de santé des habitants de la région, une hausse de la mortalité - notamment des nouveau-nés -, et un développement des maladies professionnelles et endémiques liées à la dégradation de l'environnement naturel. Près de la forge de métaux non ferreux à Szopienice, les médecins ont identifié des foyers de saturnisme prénatal, et près des forges de Dabrowa Gornicza, des cas sévères de maladies de la thyroïde chez les jeunes filles.

11 L'application très «indulgente » des sanctions - traduisant une impunité de fait en matière de responsabilité écologique - était une des conséquences logiques de la politique régionale qui favorisait les investissements industriels. Les sanctions pour le déversement dans les égouts, les émissions de poussière ou de gaz étaient symboliques et parfois même annulées. Jusqu'à la fin de l'année 1989, la sanction fiscale pour le rejet d'un kilogramme de substances toxiques à la radioactivité la plus élevée était de 3 zlotys, ce qui était l'équivalent d'un centime. Les mines n'ont jamais compensé les dégâts provoqués par une exploitation spoliatrice des gisements de houille; elles n'ont pas supporté les frais occasionnés par leur exploitation des routes, des ponts et surtout par l'ampleur de leurs déversements dans les égouts. Et aujourd'hui on sait qu'une partie des sommes consacrées à la protection de la nature a été utilisée pour la construction des bâtiments du parti communiste et des villas des fonctionnaires du précédent gouvernement... 

fonctionnement des entreprises ont été supportés par les réserves de l'environnement naturel et social. Pendant de longues années, les directeurs des entreprises, liés aux élites locales du parti, étaient au-dessus de la loi. Cette position privilégiée leur assurait l'impunité malgré la destruction de l'environnement et leur permettait une gestion prodigue de la surface utilisable, en dépit de la taille restreinte de la région. Dans une économie capitaliste, le sol urbain est un bien économique et culturel et sa valeur est précieuse. Sous le «socialisme réel ", le prix du sol destiné à des investissements industriels ne dépendait que d'une décision arbitraire des autorités politiques et ne dépassait jamais $1 \%$ des coûts d'investissement. La logique du lobby industriel destructrice pour l'environnement naturel - était simple, consistant à maximiser les effets de production sans prendre en compte les coûts écologiques et sociaux. Il n'y a encore pas longtemps, on tenait l'extraction de 190 millions de tonnes de houille dans la voïvodie pour un très grand succès, sans considérer les dégâts irréversibles subis par l'environnement naturel (affaiblissements du sol, déversements d'eaux salées dans les fleuves), ni les dégâts sanitaires (accidents du travail, qui pouvaient être mortels, maladies professionnelles).

13 Sans perdre de vue les conséquences négatives de la politique régionale, la situation écologique catastrophique de la voïvodie de Katowice et la dégradation des conditions de vie de la population peuvent s'expliquer également comme résultant d'un processus de colonisation intérieure. Ce terme, proposé par la théorie du développement dépendant, renvoie aux relations économiques, politiques et culturelles asymétriques entre une région dominante (centre politique) et les régions subordonnées (périphérie) du même pays - sans entrer dans les nombreuses nuances que distingue Alain Touraine $^{2}$. Les revenus d'une région, plutôt que d'y être réinvestis en grande partie, sont dépensés volontairement et, d'un point de vue éthique, injustement, par le centre politique et l'élite du pouvoir central. Le niveau d'investissement et les subventions aux régions défavorisées sont basés sur l'appréciation et les principes arbitraires des autorités et non sur des critères clairement définis. De surcroit, le centre, par ses préférences pour certaines branches de l'industrie, affaiblit encore l'économie des régions périphériques. Les implantations d'industries anachroniques dans la voïvodie de Katowice - comme les forges et les exploitations minières technologiquement archaïques - illustrent bien cette politique désastreuse.

14 Le terme de colonisation intérieure se réfère en effet à quatre dimensions dans les relations entre le centre et la périphérie. Dans la sphère économique, la colonisation intérieure se caractérise par un échange économique inégal et par un transfert de biens de la périphérie vers le centre du pays ; dans la sphère politique, par une asymétrie des pressions politiques entre les élites centrales d'un côté, régionales et locales de l'autre ; dans la sphère culturelle, par la "violence symbolique », la culture régionale étant éradiquée ; et dans la sphère écologique, par l'installation de technologies polluantes. Silésie - on peut constater que, depuis des dizaines d'années, cette région était, et elle est toujours, une enclave de production de matières premières, une enclave d'industries anachroniques, une région dont la culture propre a été détruite, et une région écologiquement sinistrée. C'était aussi une voïvodie où le fonctionnement du pouvoir était profondément dévoyé et où les mœurs politiques étaient pathologiques, ce qui a favorisé la dévastation de l'environnement naturel. La dégradation écologique 
progressive et souvent irréversible était accompagnée d'une répression, légale ou illégale, des "provocateurs » et de toute manifestation faisant état des conséquences négatives du "progrès industriel » de l'époque qui, tout comme la publication de données concernant l'état de l'environnement et de la santé, étaient considérés comme " antisocialistes». La crainte des autorités que le voile ne soit levé sur les données écologiques était telle qu'elles ont été presque totalement omises dans les ouvrages spécialisés, comme les annales statistiques de la voïvodie de Katowice. Les institutions et mouvements écologistes indépendants - ou associés à l'Église - et les intellectuels étaient réprimés et sanctionnés avec une intransigeance sans pareille. Seuls des organismes reconnus par l'État et la bureaucratie - comme la Liga Ochrony Przyrody (Ligue de Protection de la Nature) - étaient bien tolérés, mais ils n'avaient aucune influence réelle et leur activité demeurait marginale.

Seuls les instituts de recherche spécialisés et les centres scientifiques universitaires échappaient à cette répression. Mais les rapports et mémoires - souvent d'une grande valeur - élaborés par ces institutions n'avaient qu'une très faible audience. Ces documents ne pouvaient donc entraîner de changements dans la société, d'autant qu'une partie d'entre eux avait un caractère purement scientifique et touchait à des problèmes extrêmement spécialisés. Les nombreux appels des autorités religieuses, qui s'inquiétaient d'une exploitation spoliatrice de la région et de ses habitants, ont eu une plus grande importance. Il faut en particulier souligner le rôle de l'évêque Herbert Bednorz, qui s'est beaucoup attaché aux problèmes de la voïvodie dans le cadre de son sacerdoce.

Dans les années 1970-80, les discours des députés de la région au Parlement ont eu une certaine influence sur la prise de conscience écologique des habitants : Madame Bozena Hager-Malecka, professeur à l'Académie de médecine de Silésie, soulignait souvent l'état de santé désastreux des enfants silésiens, le problème des foyers de saturnisme prénatal endémique à Szopienice, la nécessité d'organiser des séjours de cure et de désintoxication pour les enfants et d'établir un système d'indemnisation; Tadeusz Kijonka, homme de lettres, a mis l'accent à plusieurs reprises sur le processus de dévastation de l'économie de la région et sur ses graves conséquences sociales et écologiques. Tous ces discours, souvent très dramatiques dans leur forme, n'avaient pourtant qu'une courte portée et des effets modestes - comme l'organisation de séjours de cure pour les enfants les plus menacés et l'attribution d'indemnités symboliques, grâce aux démarches de Bozena Hager-Malecka.

L'activité de nombreux mouvements écologistes - animés principalement par des étudiants et illégaux du point de vue de la législation communiste - a également eu des résultats incontestables. Leurs membres ont démontré, souvent sous une forme spectaculaire - surtout Wolnosc i Pokoj (La Liberté et la Paix) et Konfederacja Polski Niepodleglej (Confédération de la Pologne indépendante) -, la nécessité de changer l'industrie, d'arrêter certains investissements et de rétablir l'équilibre naturel. Ils ont exigé la fermeture de certaines forges et mines particulièrement nuisibles au milieu naturel. Les brochures clandestines éditées par ces organisations avaient un cercle de lecteurs limité, tout comme les rapports décrivant l'état écologique du pays et de la voïvodie parus dans des maisons d'édition indépendantes et photocopiés par les lecteurs eux-mêmes. C'est ainsi que le "Rapport Bojarski », un document bouleversant, est parvenu au public de la région. Ce document fut totalement passé sous silence par la 
presse de la voïvodie et par les autres médias contrôlés par les élites du parti communiste.

Les premiers signes d'un changement de la politique en matière écologique sont apparus dans les années 1988-89, c'est-à-dire dans la période de "démocratisation préliminaire du pays ", engagée sous la pression des grèves de mineurs dans la voïvodie de Katowice. En 1989, les députés du Conseil de la voïvodie ont considéré la région comme zone écologiquement sinistrée et ils ont rejeté le projet ministériel de restructuration de l'industrie. Ce geste d'insubordination envers le centre politique était sans précédent. Les députés soulignaient que ce projet donnait toujours la priorité aux industries extractives, énergétiques et métallurgiques, soit à des branches provoquant - pour reprendre leurs termes - « un mal écologique ». Dans cette période, les autorités régionales ont proposé au gouvernement d'introduire une " taxe écologique " destinée à la protection de l'environnement sur tous les produits dont la fabrication dégrade ou diminue le potentiel écologique de la voïvodie. Malgré des tentatives répétées, cette proposition a toujours été repoussée. Elle n'a pas non plus été acceptée par le gouvernement de Tadeusz Mazowiecki et le ministre des Finances actuel ${ }^{3}$.

En revanche, l'état catastrophique de l'environnement naturel de la voïvodie de Katowice a été pris en compte lors de la table ronde de 1989 rassemblant les autorités communistes et Solidarité. Dans le chapitre 24 du protocole de la Commission écologique on peut lire : "L'élaboration d'un plan de sauvegarde de la santé de la population jeune dans les régions écologiquement menacées est une obligation immédiate du fait d'une situation catastrophique, qui limite l'espérance de vie de la population du fait d'une situation catastrophique, qui limite l'espérance de vie de la population du pays. En Haute-Silésie, ce programme exige surtout :

- des séjours généralisés et gratuits en sanatorium pour les jeunes de moins de dix-huit ans;

- une prophylaxie pour les femmes enceintes et les enfants, aux frais des entreprises polluant le milieu naturel dans la région donnée ;

- la distribution de lait en poudre importé d'Occident au prix du lait polonais pour les enfants de moins de trois ans $"$.

21 Ce programme modeste n'a cependant pas été mis en œuvre et cet échec a renforcé l'organisation de mouvements sociaux revendicatifs, du parti vert et d'associations pour une alimentation saine et pour l'éco-développement, et a donné lieu à des joutes spectaculaires lors des élections parlementaires de juin 1989. L'analyse des programmes des partis et des organisations ayant sollicité des sièges au Parlement montre que, dans leur grande majorité, ils comportaient des déclarations écologiques bien senties, qu'ils émanent des socio-démocrates ou de la Confédération de la Pologne indépendante.

Depuis la fin 1989, des partis et mouvements écologistes se constituent dans la voïvodie de Katowice, mais ils sont encore au stade de l'élaboration de leurs programmes et de leurs statuts. Ils ont un caractère local et sont fortement fractionnés du fait des ambitions politiques de leurs leaders. Pour montrer la logique et le processus de développement du mouvement écologiste, on peut prendre l'exemple d'une des villes de la voïvodie de Katowice - la ville de Tychy. Dans cette cité, les organisations « vertes » sont assez consolidées, leurs programmes sont clairs et leurs premiers succès spectaculaires. Il semble que la ville de Tychy devienne le centre des organisations des « Verts» de la voïvodie.

Le développement du mouvement écologiste dans la ville de Tichy 

la ville a été créée ex nihilo, du temps de la Pologne communiste. Par ailleurs, Tychy devait être une ville symbolisant et matérialisant une nouvelle idéologie de l'urbanisme et de l'architecture socialiste. Enfin, Tychy devait être une extension de la ville de Karowice, fonctionnant en tant que ville satellite. en 1951, n'a été ni arbitraire ni aléatoire. Les plus importants facteurs ayant motivé ce choix ont été sa proximité de Karowice (20 kilomètres), des conditions favorables au développement du transport routier et ferroviaire - notamment pour communiquer avec d'autres centres miniers de la voïvodie -, une position centrale dans la région, des réserves de houille et d'autres matières premières, enfin des conditions climatiques et écologiques satisfaisantes. La ville, lors de sa construction, était beaucoup moins dégradée que d'autres parties de la région. Elle était protégée de la pollution industrielle grâce à des forêts qui s'étendaient à sa lisière nord. Des industries polluantes ne s'y trouvaient pas encore, à l'exception d'une usine de cellulose et de papier. Par conséquent, la pollution de l'air était relativement faible et la précipitation de la poussière n'atteignait en 1962 que $19,65 \mathrm{~g} / \mathrm{m}^{2}$ par mois (alors qu'elle était de 132,07 à Chorzow, de 116,06 à Swietochlowice, de 104,52 à Ruda Slaska, de 73,42 à Myslowice, de 62,20 à Bytom et de 44,79 à Katowice).

Aussi Tychy était-elle considérée par les médias officiels comme une «station de la Haute-Silésie », une "ville de relaxation », une " oasis verte », etc. Mais dans les années suivantes, ces atouts ont été totalement et irréversiblement détruits, du fait d'une politique «criminelle»-pour citer le rapport du groupe local des «Verts»- des autorités locales, qui ont reproduit les pires comportements mégalomanes de l'élite régionale. On a donc creusé à Tychy deux grandes mines (Piast et Ziemowit), construit la plus grande laiterie polonaise (Slask), une immense usine d'automobiles, l'usine de boissons Coca Cola, etc. Il n'est donc pas étonnant que certains quartiers de la ville surtout deux de la périphérie - aient dû supporter les conséquences d'une exploitation spoliatrice des gisements de houille et que l'on trouve dans l'air plus de quinze substances dont la quantité dépasse de plus de cent fois les normes les plus «tolérantes ». Les habitants de la ville sont effrayés par la rapidité avec laquelle la ville de Tychy rattrape - au niveau de la pollution - les villes les plus dégradées de la région. La quantité de poussière dans l'air a dépassé récemment dix fois la norme nationale (13 fois à Zabrze, 12 fois à Chorzow) ; la quantité d'azote est de cinq fois la norme, celle du benzo-alpha-pirène, considéré comme cancérigène, de huit fois, celle du plomb et du cadmium de cinq fois. Les recherches menées dans les écoles maternelles de Tychy ont montré que la quantité moyenne de cadmium dans un seul repas s'élève en hiver à 27 milligrammes, soit un dépassement de $35 \%$ de la norme - on sait que le cadmium endommage le système nerveux, osseux, circulatoire, et qu'il peut provoquer un pneumothorax.

Le plus grand danger est lié au déversement dans les fleuves des eaux salées des mines, qui contiennent des éléments radioactifs, notamment du radium 226. Chaque jour, les mines de la voïvodie versent dans la Vistule, le plus grand fleuve polonais, une quantité de 6823 tonnes de sel d'une radioactivité élevée. Avec 2604 tonnes par jour pour Piast et Czeczott et 1307 tonnes par jour pour Ziemowit, les mines de Tychy ont la plus grande responsabilité dans cette pollution. Il est difficile d'en évaluer aujourd'hui les conséquences, mais on peut déjà observer une hausse importante de la mortalité des 
nouveaux-nés (22,5\% en 1989), des avortements spontanés, des cancers, etc. On n'a pas encore entrepris de recherches scientifiques sur les conséquences de la radiation excessive dans certaines parties de la ville; jusqu'en 1985, ces recherches étaient d'ailleurs impossibles, car la documentation concernant les déversements des eaux polluées était absolument secrète.

27 Face à la dégradation de l'environnement de la ville, les médias locaux et régionaux "prenaient soin du silence », pour parler comme Ryszard Kapuscinski, un grand reporter polonais. Les autorités locales, informées de l'état de l'environnement, veillaient aussi au silence social. On ne peut donc pas s'étonner du niveau extrêmement bas de la conscience écologique des habitants, habitués aux stéréotypes de "ville de relaxation ", d'« oasis verte », etc.

Les premières initiatives sérieuses pour former un mouvement écologiste puissant datent de la fin 1988, plus précisément du 10 décembre, quand le Congrès fondateur du parti Vert s'est tenu à Cracovie, en présence des représentants de la voïvodie de Katowice. Ce parti entendait lutter - comme l'annonce son manifeste - pour un milieu naturel sain, pour le développement de la démocratie, y compris de la démocratie locale, et pour la consolidation de la paix mondiale.

29 Le 20 avril 1989, ses militants ont formé un groupe local. Ils ont rapidement conçu un programme basé sur le principe fondamental du mouvement : "Pense globalement, agis localement ». Les Verts de la ville de Tychy proposent ainsi :

30 - la transformation de la Pologne d'un pays centralisé en une confédération libre de communautés locales, prenant des décisions selon la libre volonté de leurs membres ;

31 - l'introduction du système de la démocratie directe, fondé sur des diètes civiques contrôlant leurs représentants ;

32 - le changement de la structure de l'industrie et des autres branches de l'économie, afin de diminuer leur caractère nuisible à l'environnement et leur consommation d'énergie ;

33 - l'introduction du pluralisme de la propriété et la transformation de la propriété d'État en une propriété des travailleurs ;

34 - l'abolition du militarisme, la suppression du service national obligatoire, le retrait de la Pologne du Pacte de Varsovie, l'évacuation des armées étrangères, la suppression d'une éducation militariste ;

35 - la suppression des lois autorisant une ingérence dans la vie privée des personnes et limitant leur liberté personnelle ;

36 - l'introduction de la protection des animaux et du respect de leurs droits.

37 Les Verts de la ville de Tychy ont immédiatement commencé leur activité en milieu local. Entre le 20 avril 1989 et le 15 août 1990, leurs succès ont été spectaculaires :

38 - À la suite d'une protestation, trois mines (Piast, Ziemowit et Czeczott) ont été mises en accusation devant le Tribunal administratif de la voïvodie pour pollution du milieu naturel et déversement d'eaux salées à un taux de radioactivité élevé. Les directions des mines ont été contraintes, par la décision du Tribunal, d'élaborer un programme de rétablissement de l'équilibre naturel dans un court délai.

39 - Pour la célébration du Jour de la Terre, le 23 avril 1990, les Verts ont organisé dans la ville un nettoyage général, ainsi qu'une manifestation et un happening « La terre tout entière nous appartient »; ils ont adressé une lettre de protestation au Premier ministre Tadeusz Mazowiecki et au ministre de la Protection de l'environnement. 
40 - Ils ont organisé quatre diètes locales consacrées à la «petite écologie ", à « l'éducation écologique » et à l'état de l'environnement.

41 - Ils ont protesté contre l'abattage « bestial » de cinquante quatre arbres dans le centre de la ville, où on envisageait de construire des garages pour les hauts fonctionnaires. Grâce à cette protestation, les autorités ont été obligées d'abandonner ce projet, de démolir les fondations et de planter de nouveaux arbres.

42 - Ils ont créé une section de l'Association de la protection des animaux, qui a déjà commencé son activité.

43 Le $1^{\mathrm{er}}$ avril 1990 , le groupe local de Tychy a quitté le parti Vert polonais du fait des conflits - largement commentés dans la presse régionale et centrale - qui opposaient les leaders des trois fractions concurrentes. Simultanément, les Verts de Tychy ont entrepris des démarches visant à la constitution d'une fédération regroupant la plupart des groupes écologistes existant dans la voïvodie. Le 30 juin 1990, ces initiatives ont abouti à une entente, "les Verts de la Haute-Silésie ». Sa création et ses premières activités ont attiré l'attention des autorités locales, régionales et même centrales. Le 25 août 1990, le ministre de la Protection de l'environnement est venu à Tychy, cette visite pouvant être considérée comme un événement exceptionnel pour la ville.

44 Selon ses fondateurs, l'entente « les Verts de la Haute-Silésie » est une coalition des organisations écologiques et des organisations de citoyens de la Haute-Silésie, créée en faveur de la protection de la nature dans cette région de catastrophe écologique. « En respectant le principe de la tolérance - disent les Verts - nous suivons la maxime de Saint François d'Assise: Prends soin de l'homme, de l'herbe, de l'eau et de l'air, pour que la terre n'en soit pas privée ».

Les actions des Verts en faveur de la protection de l'environnement ont bénéficié de l'approbation de la population, surtout après la parution dans la presse en 1989 et 1990 d'une série d'articles relatifs à l'écologie. Les Verts se sont présentés aux élections au conseil municipal et deux de leurs candidats ont été élus. Ils auraient sans doute eu un plus grand succès s'ils avaient présenté de plus nombreux candidats. Le groupe local de Tychy compte à présent soixante membres, et il a déjà commencé à préparer les prochaines élections parlementaires.

46 La chute du «socialisme réel » en Pologne et dans d'autres pays de l'Europe de l'Est a rendu possible la cristallisation de divers mouvements sociaux à caractère revendicatif dont l'activité était auparavant fortement limitée et réglementée par les institutions de l'État totalitaire. Parmi ces mouvements, ceux qui œuvrent en faveur de l'écologie jouent le rôle le plus important dans la transformation du système. La démocratisation a facilité leur consolidation et favorisé des initiatives visant à rétablir l'équilibre écologique. Leurs leaders comme leurs adhérents tentent de limiter l'ampleur et les conséquences de la catastrophe écologique héritée de l'époque du « socialisme réel ». On doit pourtant noter que la dégradation du milieu naturel est très profonde, souvent trop profonde pour être réparée... Et la situation économique désastreuse à laquelle le pays est confronté constitue actuellement l'obstacle le plus important à des actions efficaces en faveur de l'environnement. 


\section{BIBLIOGRAPHIE}

Conseil de la Voïvodie de Katowice, 1990, Projet de changement structurel de l'industrie à Katowice, Katowice.

Conseil de la Voïvodie de Katowice, 1990, Les orientations du changement structurel de la sidérurgie, Gliwice-Katowice.

Conseil de la Voïvodie de Katowice, Bureau des plans de développement, 1990, Le plus régional de la voïvodie de Katowice, Katowice (vol. 1 : texte ; vol. 2 : cartes).

Conseil de la Voïvodie de Katowice, Université de Silésie, 1990, Les conditions et conséquences sociales et culturelles du changement de la structure productive dans la voïvodie de Katowice (synthèse), Katowice.

Conseil de la Voïvodie de Katowice, Bureau du plan et de l'économie de la région, 1990, L'élaboration des projets de changements structuraux des entreprises dans une région industrielle traditionnelle, Katowice.

Conseil de la Voïvodie de Katowice, Bureau du plan et de l'économie de la région, 1990, La politique de changement de la structure productive de la voïvodie de Katowice. Rapport final : Les entreprises et les instruments, Katowice.

Groupe de recherches, d'innovation et d'application « Regionex », 1990, La politique de changement de la structure productive de la voïvodie de Katowice. Cahier 1 : Approche évaluative et de conception. Premiers documents. - Cahier 2 : Aspects judiciaires.

\section{NOTES}

1. Version française revue par Jeanine Cohen et Michèle Petit.

2. TOURAINE Alain, Les classes sociales dans une société dépendante. La société latinoaméricaine, Tiers Monde, vol. 16, 62, 1975.

3. Septembre 1990. (NDLR).

\section{RÉSUMÉS}

Expressions d'un processus de colonisation intérieure, l'hyperconcentration d'industries anachroniques et le fonctionnement dévoyé du pouvoir ont eu dans la voïvodie de Karowice des conséquences dramatiques sur la situation écologique et l'état de santé de la population. Les partis et mouvements écologistes qui s'y constituent depuis quelques années présentent un faible degré d'organisation et sont fortement fractionnés.

Ecological disaster, fragility of the Green movement : the Voïvodie of Katowice Significant of a process of internal colonization, both hyperconcentration of anachronistic industries and longtime inconsequent political ruling have had in the voïvodie of Katowice 
drastic consequences ont the ecological situation and on the health problems of the local population. Since a few years, ecological parties and movements which have set up in the area are still kknowing a low level of organisation and are divided into various trends.

INDEX

Mots-clés : Environnement, Industrie, écologie, Pologne, mouvements écologiques, Silésie Keywords : environment, Industry, ecology, Poland, ecological movements, Katowice, Silesia

\section{AUTEUR}

\section{MAREK S. SZCZEPANSKI}

Professeur à l'Université de Varsovie, il poursuit des recherches en sociologie urbaine, en particulier sur le développement local et les problèmes sociaux d'une région en déclin, la Haute-Silésie. 